\title{
Simultaneous Measurements of Low-Frequency Natural Electric Field at Two Neighbouring Stations
}

\author{
A. Ondrášková, S. Ševčík, P. Kostecký ${ }^{1}$, L. Rosenberg ${ }^{2}$ \\ ${ }^{1}$ Department of Astronomy, Physics of the Earth and Meteorology, Faculty of Mathematics, Physics and Informatics, \\ Comenius University, Mlynská dolina F-1, 84248 Bratislava, Slovakia, ondraskova@fmph.uniba.sk \\ ${ }^{2}$ Q.B.S., Prievozská 6, 82109 Bratislava, Slovakia, laro07@szm.com
}

\begin{abstract}
The electromagnetic eigenmodes of Earth-ionosphere global resonator known as Schumann Resonances (SR) are excited by global lightning activity. The simultaneous measurements of electric field in the ELF $(\sim 5-100 \mathrm{~Hz})$ frequency band at two observatories clearly reveal the global character of the resonator response to the excitation both in the permanent Schumann background as well as in isolated events (transients). It is shown that measurement of ELF electric field can be done with relatively simple equipment.
\end{abstract}

Keywords: Measurement of electromagnetic field, ELF frequency band, Schumann resonances, ionosphere

\section{INTRODUCTION}

$\mathrm{T}$ HE PHENOMENON of Schumann resonances (SR) - the electromagnetic eigenmodes in the resonator formed by the Earth's surface and lower ionospheric layers - was predicted by W.O. Schumann in 1952 [1] and experimentally confirmed for the first time by M. Balser and C.A. Wagner in 1960 [2]. Up to now regular observations are performed in many observatories around the world. The monitoring of SR can be considered not only as the very useful and promising tool for geophysics, but also as an interesting problem in applied electromagnetism. Both aspects are profoundly corroborated in the excellent monograph of Nickolaenko and Hayakawa [3].

We turn our attention to one particular aspect in this contribution, namely the simultaneous observations and measurements of ELF electric field component at nearby sites, which confirm the global nature of SR phenomenon. The experimental set-up for the magnetic SR component measurements, together with some results from Modra observatory was described in [4].

\section{MEASUREMENT OF ELECTRIC FIELD COMPONENT}

At the Astronomical and Geophysical Observatory of the Faculty of Mathematics, Physics and Informatics of Comenius University near Modra $\left(48.37^{\circ} \mathrm{N}, 17.27^{\circ} \mathrm{E}, 531 \mathrm{~m}\right.$ above sea level) a practically permanent monitoring of the electric field SR component has been performed since December 2001. These measurements have been made by capacitive ("ball") antenna - see Fig. 1. Summary of results from more than 4 years of monitoring was published in [5].

The SR eigenmodes are TEM-type waves, therefore the dominant electric field component is vertical (radial), at least immediately above the Earth's surface. The receiving "ball" type antenna is omnidirectional. The upper electrode (conductive ball) has a capacitance of about $50 \mathrm{pF}$ with respect to the second electrode made from grounding plates buried approx. $60-80 \mathrm{~cm}$ below the surface, encircling the antenna base. The voltage between electrodes is amplified by a very high input impedance amplifier (with gain of about 82 $\mathrm{dB}$ at $10 \mathrm{~Hz}$ ), subsequently fed to the 16-bit $\mathrm{ADC}$, digitized and stored (short description of the receiving equipment is given at the end of Section 4). The antenna internal impedance can be considered as purely capacitive, therefore the input voltage of the amplifier can be expressed as:

$$
U_{\text {in }}(\omega)=E_{r} \cdot h_{\text {eff }} \frac{i \omega R C_{a n t}}{1+i \omega R\left(C_{a n t}+C_{\text {in }}\right)}
$$

where $\omega$ stands for the angular frequency, $h_{\text {eff }}$ is the effective height of antenna (in our case, it is its geometrical height $(5 \mathrm{~m})$ reduced by an empiric factor of approx. 0.6), $R$ is the total resistance between active electrode and ground, including the insulation resistance of antenna mast and cable, amplifier input resistance, etc., $C_{\text {ant }}$ represents the active electrode-toground capacitance and $C_{\mathrm{in}}$ is the amplifier input capacitance together with the capacitance of input circuit.

In our case, we can estimate: $C_{\text {ant }} \sim 50 \mathrm{pF}, C_{\text {in }} \sim 20 \mathrm{pF}, R \sim$ $1000 \mathrm{MOhm}$ (under normal conditions). The characteristic frequency of input circuit is about $15 \mathrm{~Hz}$, which is in near accordance to experimental value. By appropriate adjusting of amplifier frequency response, the overall flat response can be achieved. Because the principal part of total input resistance $R$ (which is difficult to estimate) is the antenna mast resistance, then during heavy rain, snow or icing the input circuit transfer function in SR frequency range substantially decreases and the SR signal disappears in the noise background.

The average amplitude of the electric field SR component can be estimated from the amplitude of ADC input voltage. For ordinary "Schumann background" the signal spans about $0.25 \mathrm{~V}$ (peak-to-peak) that corresponds to approx. $25 \mu \mathrm{V}$ amplifier input voltage. Taking into account the input circuit transfer factor and the antenna effective height, the estimate for the electric field intensity is $6-8 \mu \mathrm{V} \cdot \mathrm{m}^{-1}$. Dividing by free-space impedance $\left(Z_{0}=120 \pi \mathrm{Ohm}\right)$ and using the vacuum permeability $\mu_{0}$, the estimate for magnetic induction amplitude of Schumann background is $B \sim 0.03 \mathrm{pT}$, which is in accordance with the results of SR magnetic components observation [4].

\section{SCHUMANN BACKGROUND AND TRANSIENTS}

The natural SR signal usually seems to be "noise-like". 
Nevertheless, the Fourier spectrum of the signal accumulated for some tens of seconds or minutes clearly shows the quasiregular pattern of peaks at characteristic mode frequencies. The peak frequencies, $Q$-factors and amplitudes of individual modes exhibit the variations in various time scales (from hours to years). The most important sources of these variations are the seasonal migration of lightning activity foci on Earth, the changes of the ionosphere structure and the influence of solar activity.

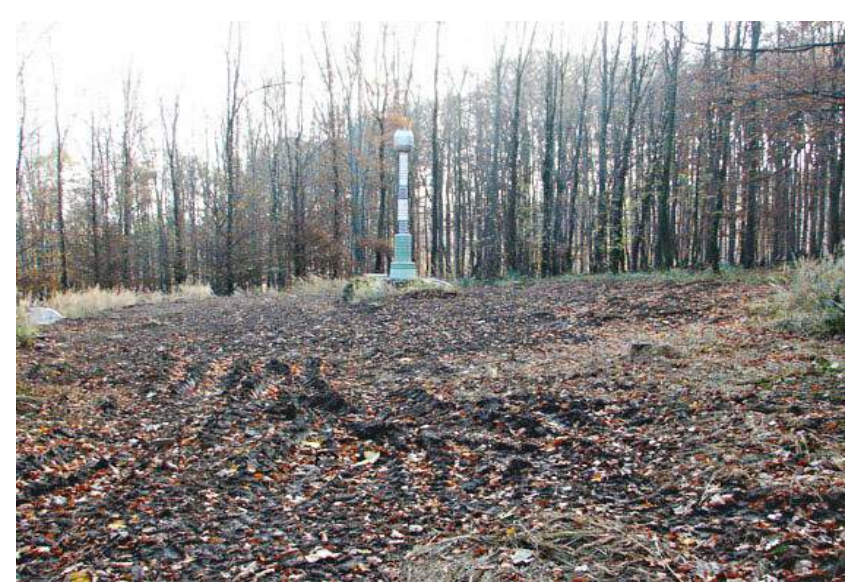

Fig.1 The capacitive antenna for monitoring Schumann resonance electric field component at AGO. The amplifier and analogue filters are placed in a small screened box directly at the bottom near the antenna base.

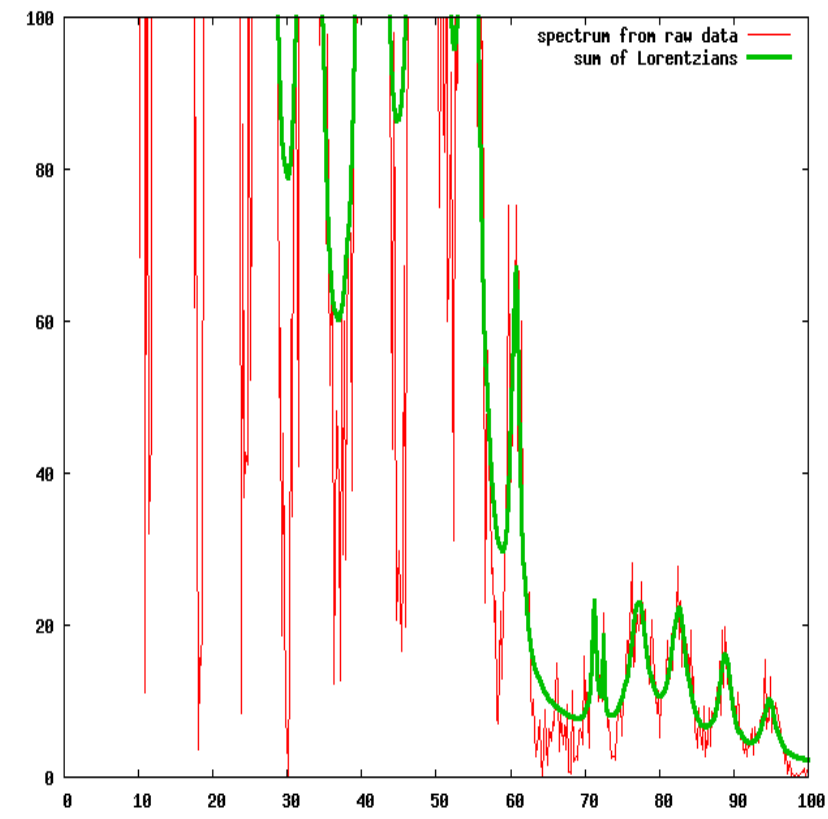

Fig.2 Raw spectrum of SR transient (thin line) and the same spectrum fitted by the weighted sum of Lorentz functions (thick line) processed in short-time $(1.28 \mathrm{~s})$ data set. The characteristic pattern of higher SR modes $(60-100 \mathrm{~Hz})$ is clearly visible (lower modes are out of range due to linear vertical scale). Only if the resonator is excited by a single, extraordinary intense lightning discharge, the higher modes can appear in the short-time spectra shown here.
It is well known that the majority of global lightning activity is concentrated into three tropical areas (equatorial Africa, Indonesia and Amazonia). The great number of lightning discharges scattered in space and time excite the quasi-permanent SR field oscillations quoted as "Schumann background". From time to time, spatially isolated huge discharges can excite the Earth-ionosphere resonator in such a strong manner, that the higher modes can be distinguished in the spectra obtained from short (several seconds) time series. These phenomena are named "Q-bursts" and manifest themselves usually as "double-spikes" of bipolar time pattern, separated by some tenths of a second. Their amplitude exceeds in most cases the average value of "Schumann background" by a factor of ten. The coherence of their excitation sources allows (at least in principle) to reveal the spatial pattern of E.M.field for SR modes when transient event is detected at more observatories distributed over the Earth's surface. For most of the time, higher SR modes $(f>40 \mathrm{~Hz})$ are buried in the Schumann background spectra, because the corresponding wavelength is generally several thousand $\mathrm{km}$ and multiple sources (discharges) are non-coherent at this spatial scale. Typical "higher-modes" portion of transient spectrum can be seen in Fig.2.

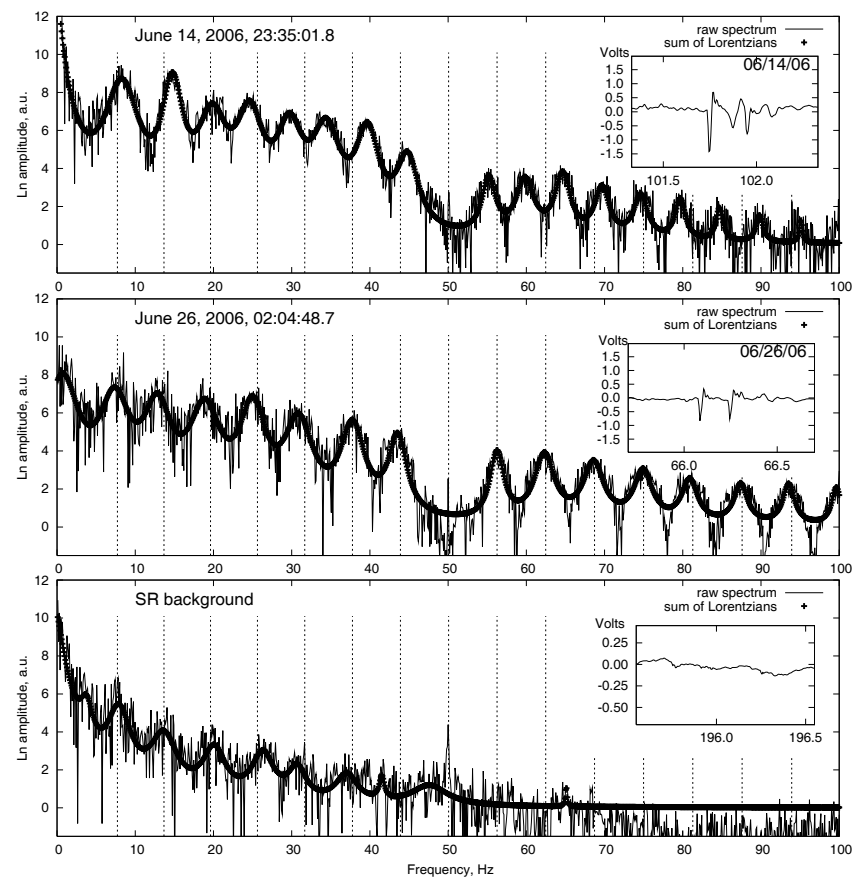

Fig.3 The examples of raw short-time spectra of transient signals (two subgraphs from above) and the spectrum of Schumann background signal (lowest subgraph). All spectra obtained by DFT are shown by thin lines, the results of non-linear least-square fitting by sum of Lorentz-like functions are depicted by thick lines. The vertical dotted lines indicate the average peak frequencies of Schumann modes. The apparent "holes" around $50 \mathrm{~Hz}$ are artifacts of analogue notch filters suppressing the public line hum.

In Fig.3, two examples of short-time spectra from signal sequences $10.24 \mathrm{~s}$ long containing transients (in upper right rectangles, the $1 \mathrm{~s}$ parts of time recordings) are depicted. The upper subgraph shows the usual form of transient event ("Q- 
burst"). The first (leading) bipolar spike is excited by lightning discharge directly, the second spike - on its way around the globe - is slightly attenuated and widened in time by frequency dispersion. The interspike interval (around $0.13-$ $0.15 \mathrm{~s})$ corresponds to the wavefront traveling time for one Earth's orbit.

The second subgraph from above shows the peculiar transient possessing the same interspike interval, but without any sign of attenuation and dispersion. Transients of this type (of obscure origin up to now) are observed frequently, which definitely cannot be attributed to pure chance [6].

The lowest subgraph shows the spectrum of time sequence of pure Schumann background. We can clearly see that higher Schumann modes (for index $n>6$, frequency $f>50 \mathrm{~Hz}$ ) are totally absent. The wavelengths of higher Schumann modes (taking into account the phase velocity about $0.75-0.8 \mathrm{c}$, due to ionospheric plasma properties) are comparable to spatial dimensions of principal thunderstorm foci.

\section{SiMULTANEOUS OBSERVATIONS FROM MODRA AND NAGYCENK}

All three principal components of SR field (vertical electric and two orthogonal horizontal magnetic components) have been regularly monitored at Nagycenk observatory in Hungary, operated by Geophysical and Geodetical Research Institute of the Hungarian Academy of Sciences $\left(16.71^{\circ} \mathrm{E}\right.$, $\left.47.63^{\circ} \mathrm{N}\right)$. The experimental set-up for electric field measurements is very similar to the equipment used in Modra. The straight-line distance between both observatories is about $92 \mathrm{~km}$. By courtesy of Dr. József Bór from Nagycenk observatory, we have had the possibility to compare results of SR electric field measurements at both sites. Moreover, the identification of numerous Q-bursts has been successful. In Fig. 4 both records of an event from June 26, 2006 are shown as an example. High correlation (not only for the transient itself, but also for the "background signals") is a clear evidence that the observations record the global SR field pattern. Minor differences in the time patterns can be attributed to local effects, and, predominantly, to slightly different frequency responses of receiving tracts in both cases.

Here is a brief description of the receiving equipment for electrical component SR field monitoring used at Modra observatory (AGO):

The amplifier (situated immediately at antenna base) has a symmetrical input bridge circuit with a twin vacuum electrometric tube as an active element. The rest of amplifier circuitry is completely solid-state, with a complementary (symmetric) output driving twisted-pair cable (of $\sim 150 \mathrm{~m}$ length) to $\mathrm{ADC}$ and $\mathrm{PC}$ for subsequent data processing. The experiments with high impedance FET operational amplifier at the input have provided analogical results, but they were more vulnerable to close atmospheric discharges.

The total gain of amplifier is about $82 \mathrm{~dB}$ at $10 \mathrm{~Hz}$. The broadband, very low-frequency noise (below the SR frequency range) is suppressed by sixth order active high-pass (corner frequency $\sim 4.5 \mathrm{~Hz}$ ). Analogical suppression of public line hum is ensured by a couple of $50 \mathrm{~Hz}$ notches. After analogue processing, the signal is digitized at sampling frequency 200 $\mathrm{Hz}$ by 16-bit separate ADC and, finally, stored in PC for next spectral processing.
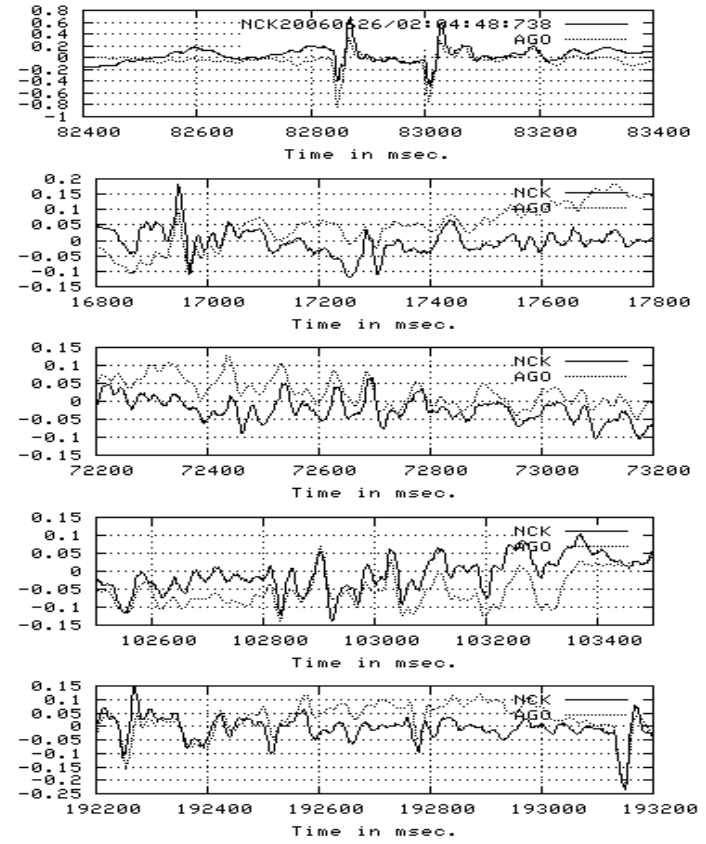

Fig.4 Example of simultaneous record of Q-burst (in the electric field component) from June 26, 2006, 02:04:48.8 UT. The signal from observatory in Modra (dashed line) and signal from Nagycenk observatory (solid line) represent the simultaneous recording of the electric field component: The upper graph: $1 \mathrm{sec}$ long record covering the event itself. The vertical scale is (in all graphs) in volts at the ADC input. The lower graphs (note the different vertical scale) show the "Schumann background" signal. The second and the third graph from the top are the 1-second intervals before the event, last two show the equal length intervals after the event. Note the different vertical scales on the graphs.

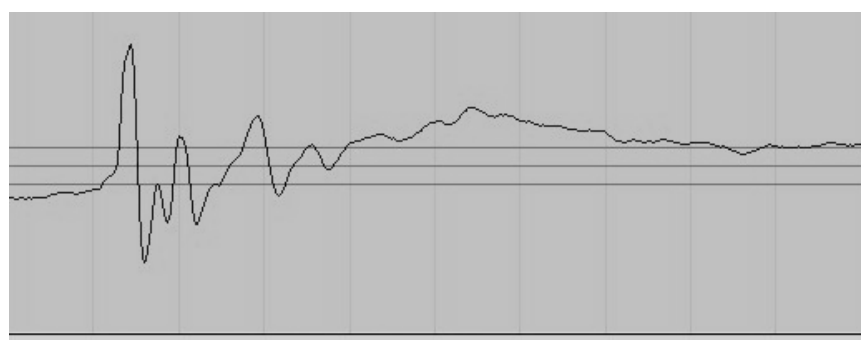

Fig.5a The record of transient event from January 3, 2007, 07:20:28 UT (electric field component) made at Nagycenk Observatory, Hungary.

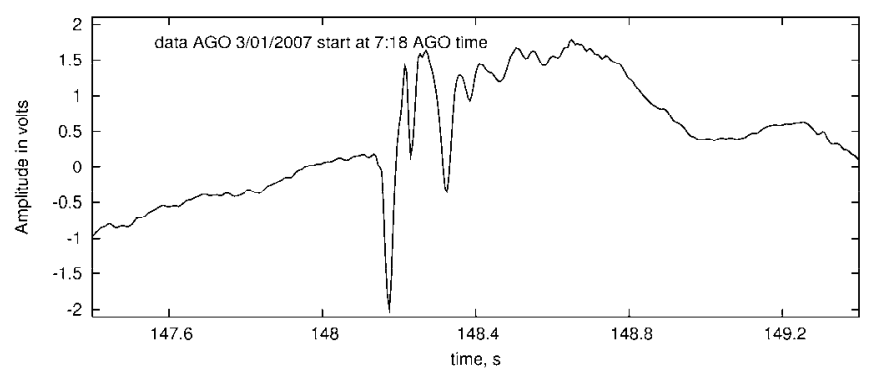

Fig.5b The same transient event recorded at Modra Observatory (AGO) also in electric field component (the time scale is different with respect to the above graph). Apparent polarity inversion of record is due to instrumental effect. 
For more distant sites of observations, the correlation (similarity) between SR signal time sequences deteriorates in some degree, due to the spatial distribution ("angular patterns") difference of distinctive eigenmodes. Nevertheless, some degree of correlation persists even for antipodal sites.

As an example, Fig.6 shows the time record of the same transient (as in Figs.5a,b) from Mitzpe Ramon Observatory (Negev desert, Israel) in magnetic E-W component.

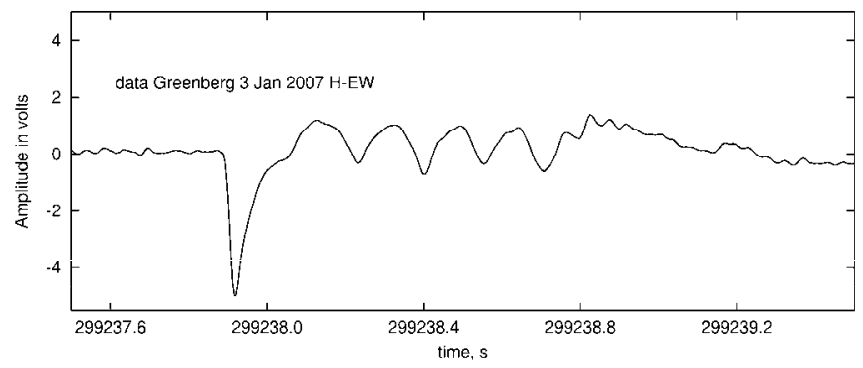

Fig.6 Magnetic field component record (E-W direction) of the same transient event as in Figs.5a,b, recorded at Mitzpe Ramon Observatory, Israel (distance from AGO: $2753 \mathrm{~km}$ ). The data were provided by courtesy of Dr. Eran Greenberg, Department of Geophysics, Tel Aviv University. Unfortunately, simultaneous data of electric field component were accidentally not recorded.

\section{CONCLUSION}

The measurements of low-frequency A.C. vertical electric field over the Earth's surface can be done with relatively simple equipment. Results of long-time observation series confirm that the signals analyzed are clearly of natural and global origin. The accumulation of data covering SR transient events can be very helpful for studies of the Earth-ionosphere global resonator properties. Their changes are reflecting the influences of Earth's internal forces and also of cosmic origin, e.g. solar activity $-[7]$.

\section{ACKNOWLEDGMENT}

The authors gratefully acknowledge support by grant No. 1/2033/05 from the Scientific Grant Agency VEGA. We also gratefully acknowledge help from Dr. József Bór from the Geophysical and Geodetical Research Institute of Hungarian Academy of Sciences, Sopron, Hungary, for regularly providing the measured data obtained at Nagycenk observatory.

\section{REFERENCES}

[1] Schumann, W.O. (1952). On the free oscillations of a conducting sphere which is surrounded by an air layer and an ionosphere shell (in German). Z. Naturforschung 7a, 149-154.

[2] Balser, M., Wagner, C.A. (1960). Observation of earthionosphere cavity resonances. Nature 188, 638-641.

[3] Nickolaenko, A.P., Hayakawa, M. (2002). Resonances in the earth-ionosphere cavity. Dordrecht, NL: Kluwer Academic Publishers.

[4] Ondrášková, A., Ševčík, S., Rosenberg, L., Kostecký, P., Turňa, L., Kohút, I. (2005). The detection of magnetic field component of Schumann resonances using search coil sensors at Modra observatory. In: Measurement 2005: Proceedings of the 5th International Conference on Measurement, 15-19 May 2005 (pp. 506-509). Bratislava, Slovakia: Institute of Measurement Science, SAS.

[5] Ondrášková, A., Kostecký, P., Ševčík, S., Rosenberg, L. (2007). Long-term observations of Schumann resonances at Modra observatory. Radio Sci. 42(2), RS2S09.

[6] Ondrášková, A., Kostecký, P., Ševčík, S., Rosenberg, L. (2008). Peculiar transient events in the Schumann resonance band and their possible explanation. J. Atm. Sol.-Terr. Phys. 70(6), 937-946.

[7] Roldugin, V.C., Maltsev, Y.P., Vasiljev, A.N., Shvets, A.V., Nickolaenko, A.P. (2003). Changes of Schumann resonance parameters during the solar proton event of 14 July 2000. J. Geophys. Res. 108(A3), 1103-1109. 